\title{
Stress intensity factor for the interaction between a straight crack and a curved crack in plane elasticity
}

\begin{abstract}
Formulation in terms of hypersingular integral equations for the interaction between straight and curved cracks in plane elasticity is obtained using the complex variable functions method. The curved length coordinate method and a suitable numerical scheme are used to solve such integrals numerically for the unknown function, which are later used to find the stress intensity factor, SIF.
\end{abstract}

Keyword: Hypersingular integral equation; Complex variable function method; Curved length coordinate method; Stress intensity factor 